\title{
Antike Quellen
}

\section{Septuaginta}

\begin{tabular}{|c|c|c|c|}
\hline \multicolumn{2}{|l|}{ Genesis } & \multicolumn{2}{|c|}{ Deuteronomium } \\
\hline 2,24 & 694 & 5,16 & 853 \\
\hline 12 & 904 & $5,17-19$ & 753 \\
\hline 12,3 & 896 & 5,21 & 753 \\
\hline 15 & $900,904,906$ & $27-30$ & 907 \\
\hline 15,5 & 686 & 30,14 & 818 \\
\hline 15,6 & 237,924 & 32,12 & 687 \\
\hline $16-21$ & 481 & 32,35 & 690 \\
\hline 17,8 & 920 & 32,43 & 689 \\
\hline 18 & 904 & & \\
\hline 18,10 & 686 & Richter & \\
\hline 18,14 & 686 & 8,12 & 302 \\
\hline 18,18 & 896 & 13,23 & 821 \\
\hline 21,10 & 567 & 20,45 & 301 \\
\hline 21,21 & 688 & 1 Samuel & \\
\hline 25,23 & 686 & 6,12 & 326 \\
\hline 30,11 & 205 & 11,3 & 747 \\
\hline 42,21 & 337 & & \\
\hline \multirow[t]{2}{*}{50,25} & 782 & 1. Könige & \\
\hline & & 19 & 979 \\
\hline Exodus & & 19,8 & 979 \\
\hline 4,10 & 934 & & \\
\hline 19,13 & 782 & 2. Könige & \\
\hline 20,12 & 853 & 9,27 & 302 \\
\hline $20,13-15$ & 753 & 23,17 & $749-750$ \\
\hline 20,17 & 753 & & \\
\hline 24,8 & 782 & Nehemia & \\
\hline 25 & 468 & 9 & 907 \\
\hline 32 & 903 & & \\
\hline 32,6 & 389,903 & ljob & \\
\hline 33,19 & 688 & 35,16 & \\
\hline \multicolumn{2}{|l|}{ Levitikus } & Psalter & \\
\hline \multirow{2}{*}{19,18} & 753 & 4,5 & 756 \\
\hline & & 8,7 & $438,699,976$ \\
\hline \multirow{2}{*}{\multicolumn{2}{|c|}{ Numeri }} & 17,50 & 689 \\
\hline & & 18,10 & 598 \\
\hline 16,5 & 359 & 18,49 & 689 \\
\hline $16,41-56$ & 389 & 14,34 & 22 \\
\hline \multirow[t]{2}{*}{$25,1-9$} & 389 & 50,6 & 420,685 \\
\hline & & 50,18 & 821 \\
\hline
\end{tabular}

๖ Open Access. $\odot 2020$ Christoph Heilig, publiziert von De Gruyter. @) BY Dieses Werk ist lizenziert unter der Creative Commons Attribution 4.0 International Lizenz.

https://doi.org/10.1515/9783110670691-020 


\begin{tabular}{|c|c|c|c|}
\hline 60,8 & 598 & Hosea & \\
\hline 61,13 & 691 & 2,1 & 687 \\
\hline $67,18-19$ & 934 & 2,24 & 687 \\
\hline 109 & 976 & 13,14 & 438 \\
\hline 117,1 & 689 & & \\
\hline \multirow[t]{2}{*}{144,15} & 361 & Joel & \\
\hline & & 3,5 & 633,690 \\
\hline \multicolumn{2}{|l|}{ Proverbia } & Jona & \\
\hline 24,12 & 691 & $1,1-3$ & 470 \\
\hline $25,21-22$ & 817 & & \\
\hline \multirow[t]{2}{*}{25,22} & 691 & Habakuk & \\
\hline & & 1,5 & $5-6$ \\
\hline \multicolumn{2}{|l|}{ Hoheslied } & $2,2-3$ & 920 \\
\hline 5,3 & 759 & 2,4 & $699,919-920$ \\
\hline \multicolumn{2}{|l|}{ Jesaja } & \multicolumn{2}{|l|}{ Haggai } \\
\hline 1,9 & 665,687 & 2,16 & 425 \\
\hline $6,9-10$ & 851 & \multicolumn{2}{|l|}{ Sacharja } \\
\hline 8,14 & 189 & \multirow{2}{*}{8,23} & \multirow{2}{*}{899} \\
\hline $10,22-23$ & 687 & & \\
\hline 11,10 & 689 & \multicolumn{2}{|l|}{ Judith } \\
\hline 25,8 & 438 & \multirow[t]{2}{*}{$15,2-3$} & \multirow[t]{3}{*}{301} \\
\hline 27,9 & 437 & & \\
\hline 28,16 & $689-690,818$ & Weisheit & \\
\hline 29,22 & 434 & $8,19-20$ & 651 \\
\hline $40-55$ & 907 & 11,21 & 425 \\
\hline $40-66$ & 907 & 13,6 & 656 \\
\hline 40,5 & 908 & 14,19 & 656 \\
\hline 40,13 & 359,829 & 19,18 & 425 \\
\hline 43,19 & 659 & & \\
\hline 45,14 & 899 & Tobit & \\
\hline 45,23 & $685,691,698,850$ & 1,6 & 330 \\
\hline 49,2 & 435 & & \\
\hline 52,7 & 634,637 & Sirach & \\
\hline 52,15 & 605,687 & 12,15 & 598 \\
\hline $52,20-21$ & 690 & 22,23 & 598 \\
\hline \multirow{2}{*}{64,1} & \multirow{2}{*}{908} & 25,21 & 656 \\
\hline & & 40,7 & 598 \\
\hline \multicolumn{2}{|l|}{ Jeremia } & 41,13 & 598 \\
\hline 1,5 & 434 & 44,11 & 598 \\
\hline 2,12 & 222 & 46,9 & 598 \\
\hline 3,5 & 598 & \multirow{2}{*}{\multicolumn{2}{|c|}{ 2. Makkabäer }} \\
\hline \multirow{2}{*}{9,4} & $289,362-363$ & & \\
\hline & & 2,18 & 361 \\
\hline Ezechiel & & 6,23 & $651-652$ \\
\hline 1 & 934 & 7,19 & 729 \\
\hline
\end{tabular}


3. Makkabäer

$5,5 \quad 729$

$6,31 \quad 652$

$7,5 \quad 652$
4. Makkabäer

16,22 236

\section{Neues Testament}

Das Evangelium nach Matthäus

Werk

1,18

1,24

$6-15,33,82,130,454$

408

783

2,7

392

2,12

464

2,13

380

2,14-19

4,6

4,11

5,23-24

5,25

6,8

6,16

6,33

7,9-10

7,28

8,3

8,4

8,9

8,15

8,18

8,21

8,27

8,34

9,15

9,18-26

9,25

10,5

10,9

10,10

11,1

11,11

12,10

12,29

13,10-11

13,45-46

13,46
464

782

326

406-407

429

409

422

406

709

290, 362

789

789

783

326

782

648

783

776

430

786

395

787, 789

789

788

786-787

354

704

407

190

319,321
172, 201, 243-245
$13,49 \quad 434$

$14,9 \quad 782-783$

$14,18 \quad 782$

15,2 422-423

$15,14 \quad 681,817$

$15,23 \quad 523$

$15,24 \quad 649$

15,30-31 339

$15,35 \quad 789$

$16,6 \quad 782$

$16,20 \quad 782,789$

$16,26 \quad 706$

$17,9 \quad 782$

$17,15 \quad 344$

$17,16 \quad 523$

$17,18 \quad 783$

18,6 762

$18,25 \quad 782$

$19,7 \quad 782$

19,21 321

$21,6 \quad 782$

$21,38 \quad 695$

22,9-10 784

$23,26 \quad 407$

$25,40 \quad 579$

$26,19 \quad 783$

$26,26 \quad 392$

$26,34 \quad 408$

$26,36 \quad 454$

$26,38 \quad 454$

$26,41 \quad 741$

$26,45 \quad 454$

$26,75 \quad 408$

$27,10 \quad 783$

$27,14 \quad 339,523$

$27,44 \quad 762$

$27,49 \quad 381$

28,8-11 789 


\begin{tabular}{|c|c|c|c|}
\hline 28,10 & 852 & 9,42 & 762 \\
\hline 28,16 & 783 & 10,3 & 782 \\
\hline 28,19 & 379 & 10,49 & 782 \\
\hline \multirow[t]{2}{*}{28,20} & 782 & 11,1 & 417 \\
\hline & & $11,4-6$ & 782 \\
\hline \multicolumn{2}{|c|}{ Das Evangelium nach Markus } & 11,6 & 783 \\
\hline Werk & $6-15,33,82$ & 11,19 & 424 \\
\hline 1,11 & 353 & 12,7 & 695 \\
\hline 1,13 & 326 & 12,40 & 826 \\
\hline 1,27 & 783 & 13 & 524 \\
\hline 1,31 & 326 & $13,9-10$ & 407 \\
\hline 1,43 & 789 & 13,14 & 524 \\
\hline 1,44 & 783 & 13,21 & 524 \\
\hline 2,7 & 649 & 13,26 & 524 \\
\hline 2,12 & $338-339$ & 13,17 & 524 \\
\hline 2,19 & 430 & 13,30 & 411, 413 \\
\hline 2,20 & 524 & 14,12 & 425 \\
\hline 3,10 & $338-339$ & 14,16 & 783 \\
\hline 3,11 & 424 & 14,22 & 392 \\
\hline 3,14 & 670 & 14,30 & 408 \\
\hline 3,27 & 407,524 & 14,32 & 454 \\
\hline 4,12 & 373 & 14,38 & 741 \\
\hline 4,35 & 782 & 14,72 & 408 \\
\hline 4,37 & 339 & 15,5 & 339 \\
\hline 4,41 & 783 & 15,23 & 762 \\
\hline 5,8 & 789 & 15,41 & 326 \\
\hline 5,10 & 784 & 16,4 & 762 \\
\hline 5,15 & 295 & 16,6 & 362 \\
\hline $5,21-43$ & 786 & 16,8 & 301,789 \\
\hline 5,43 & 782,789 & 16,11 & 362 \\
\hline 6,8 & 789 & 16,14 & 362 \\
\hline 6,9 & 759,789 & & \\
\hline 6,14 & 354 & \multicolumn{2}{|c|}{ Das Evangelium nach Lukas } \\
\hline 6,26 & 782 & Werk & $6-15,33,82,341,394$ \\
\hline 6,27 & 783 & 1,30 & 177 \\
\hline 6,39 & 782 & 1,31 & 381 \\
\hline 7,34 & 344 & 1,42 & 381 \\
\hline 7,36 & 237,782 & 1,64 & 327 \\
\hline 8,3 & 177 & 2,21 & 381,409 \\
\hline 8,6 & 789 & 2,26 & 408 \\
\hline 8,15 & 782 & $2,36-38$ & 336 \\
\hline 8,30 & 782,789 & 3,13 & 786 \\
\hline 9,9 & 782 & 4,10 & 782 \\
\hline 9,25 & 783 & 4,27 & 648 \\
\hline 9,26 & 354 & 4,36 & 783 \\
\hline 9,27 & 354 & 4,39 & 326 \\
\hline 9,34 & 162 & 5,14 & 783,789 \\
\hline
\end{tabular}




\begin{tabular}{|c|c|c|c|}
\hline 5,23 & 295 & 20,16 & 761 \\
\hline 7,8 & 783 & 20,47 & 826 \\
\hline $7,37-45$ & 433 & 21,9 & 407 \\
\hline 7,45 & $432-433$ & 22,6 & 326 \\
\hline 7,47 & 226 & 22,13 & 783 \\
\hline 8,25 & 783 & 22,15 & 381,409 \\
\hline 8,29 & 789 & 22,19 & 392 \\
\hline 8,31 & 784 & 22,20 & 392,402 \\
\hline 8,42 & 283 & 22,28 & 596 \\
\hline 8,49 & 288 & 22,30 & 851 \\
\hline 8,51 & 648 & 22,36 & 826 \\
\hline 8,53 & 177 & 22,32 & 380 \\
\hline 8,55 & 786 & 22,39 & 454 \\
\hline 8,56 & 789 & 22,61 & 408 \\
\hline 9,3 & 759,789 & 24,5 & 362 \\
\hline $9,14-15$ & 782 & 24,9 & 381 \\
\hline 9,21 & 782,789 & 24,11 & $378-379$ \\
\hline 9,42 & 783 & 24,21 & 432 \\
\hline 9,59 & 406 & 24,23 & $362,378,380-381$ \\
\hline 9,61 & 218,406 & & \\
\hline 10,5 & 406 & \multicolumn{2}{|c|}{ Das Evangelium nach Johannes } \\
\hline 11,5 & 765 & Werk & $6-15,25,33,82,322,394$ \\
\hline 11,10 & 648 & 1,15 & 348 \\
\hline 11,11 & 640 & 1,32 & 365 \\
\hline 11,19 & 224 & 1,45 & 381 \\
\hline 12,1 & 782 & 1,46 & 381 \\
\hline 12,7 & 177 & 1,48 & 381,409 \\
\hline 12,11 & 422,493 & 3,16 & 377 \\
\hline 13,7 & 432 & $4,1-3$ & $650-651$ \\
\hline 13,23 & 704 & 4,4 & 663 \\
\hline 13,25 & 423 & 4,8 & 322 \\
\hline 14,13 & 422 & 4,31 & 415 \\
\hline 14,14 & 222,435 & 4,49 & 408 \\
\hline 14,22 & 784 & 5,2 & 348 \\
\hline 14,26 & 747 & 5,18 & 643 \\
\hline 14,28 & 407 & 5,25 & 696 \\
\hline 14,31 & 407,743 & 5,45 & 293,295 \\
\hline 14,32 & 431 & 6,17 & 322 \\
\hline 15,32 & 351 & 6,26 & 403 \\
\hline 16,4 & 351 & $6,39-40$ & $851-852$ \\
\hline 17,9 & 786 & 6,51 & 681,817 \\
\hline 17,10 & 786 & 7,15 & 363 \\
\hline 17,25 & 407 & 7,17 & 681,817 \\
\hline 18,8 & 704 & 7,30 & 322 \\
\hline 18,9 & 747 & 7,31 & 709 \\
\hline 18,40 & 287 & 7,39 & 592 \\
\hline $19,32-34$ & $782-783$ & 7,50 & 403 \\
\hline
\end{tabular}




\begin{tabular}{|c|c|c|c|}
\hline 7,51 & 407 & 2,20 & 408 \\
\hline 8,30 & 322 & $2,24-25$ & 119 \\
\hline 8,39 & 747 & 2,44 & 361 \\
\hline 8,44 & 423 & 4,18 & 789 \\
\hline 8,58 & 408 & 4,27 & $221-222$ \\
\hline 9,1 & 434 & 5,14 & 651 \\
\hline 9,8 & 403 & 5,26 & 745 \\
\hline 9,18 & 523,725 & 5,28 & 789 \\
\hline 9,22 & 322 & 5,40 & 789 \\
\hline 10,10 & 649 & $5,40-41$ & 338 \\
\hline $11,6-7$ & 393 & 7,2 & 408 \\
\hline 11,13 & 322 & 7,11 & 282 \\
\hline 11,18 & 348 & 7,16 & 762 \\
\hline 11,19 & 322 & 7,27 & 184 \\
\hline 11,30 & 322 & 7,35 & 319 \\
\hline 11,44 & 322 & 7,44 & 786 \\
\hline 11,51 & 523 & 7,47 & 161 \\
\hline 11,53 & 743 & 9 & 468 \\
\hline 11,57 & 322 & 9,6 & 783 \\
\hline 12,10 & 743 & 9,11 & 343 \\
\hline 12,40 & 851 & $9,24-25$ & 575 \\
\hline 13,9 & 381 & 9,26 & 725 \\
\hline 13,19 & 409 & 9,27 & 5 \\
\hline $14-15$ & 782 & 9,30 & 465 \\
\hline 14,29 & 408 & 10,33 & 783 \\
\hline 14,31 & 782 & 10,42 & 789 \\
\hline 15,14 & 782 & 10,48 & 783 \\
\hline 15,17 & 782 & 10,84 & 597 \\
\hline 17,5 & 409 & $11,25-26$ & 465 \\
\hline 17,22 & 291 & $11,27-30$ & 465 \\
\hline 18,22 & 427 & 11,28 & 420 \\
\hline 18,23 & 523 & 12,1 & 339 \\
\hline 19,22 & 291 & 13,11 & 327 \\
\hline 19,28 & 291 & 13,15 & 6 \\
\hline 19,41 & 348 & 13,16 & 6 \\
\hline 20,18 & 788 & 13,40 & 5 \\
\hline 20,24 & 396 & 13,41 & 5 \\
\hline 20,31 & 670 & 13,47 & 782 \\
\hline 21,22 & 429 & 13,48 & 783 \\
\hline 21,23 & 429 & 14,1 & 286 \\
\hline \multirow[t]{2}{*}{20,31} & 25 & 14,9 & 725 \\
\hline & & 14,27 & 291 \\
\hline \multicolumn{2}{|c|}{ Die Apostelgeschichte } & 15 & $505-506$ \\
\hline Werk & $6-15,33,36,124$ & 15,2 & 783 \\
\hline 1,2 & 782 & 15,3 & 5 \\
\hline 1,4 & 789 & 15,5 & 789 \\
\hline 1,6 & 704 & 15,12 & 5 \\
\hline
\end{tabular}




\begin{tabular}{|c|c|c|c|}
\hline 15,14 & 5 & 28,23 & 783 \\
\hline 15,22 & 312 & 28,31 & 5 \\
\hline 15,24 & 782 & & \\
\hline 15,27 & 312 & \multicolumn{2}{|c|}{ Der Brief an die Römer } \\
\hline 15,30 & 26,312 & Brief & $3,26,154-156,341,609-612$, \\
\hline 15,31 & 312 & & $740-743$ \\
\hline 15,32 & 312 & 1,1 & 4, 435 \\
\hline $16,6-10$ & $530-533$ & $1-5$ & 860,906 \\
\hline 16,10 & 5 & 1,3 & 435 \\
\hline 16,11 & 531 & 1,4 & 435 \\
\hline 16,18 & 789 & 1,8 & 405 \\
\hline 16,23 & 789 & 1,9 & $344-345$ \\
\hline 16,29 & 642 & $1,9-10$ & 427 \\
\hline 16,40 & 5,532 & 1,10 & $407,704,730-731,738$ \\
\hline $17,1-14$ & & 1,11 & $472,738,742-743,831$ \\
\hline 17,5 & 844 & 1,12 & 205 \\
\hline 17,15 & 401 & 1,13 & $327,331,740-741,766$ \\
\hline $17,24-31$ & 975 & $1,13-15$ & $741-743$ \\
\hline 17,26 & 783 & 1,16 & 405,742 \\
\hline 17,30 & 789 & $1,18-24$ & $619-620,623,627$ \\
\hline 17,32 & 636 & 1,20 & $221-222,369,434$ \\
\hline 18,2 & 786 & 1,21 & 628 \\
\hline 20,6 & 165 & 1,25 & 389 \\
\hline \multirow[t]{2}{*}{20,7} & $161-162,164-165,172-175$ & 1,32 & $231-232,643$ \\
\hline & $380,383,460-461$ & 2,1 & 318 \\
\hline 20,13 & 786 & 2,2 & 729 \\
\hline 21,19 & 5 & 2,3 & 708,729 \\
\hline 21,20 & 329 & 2,4 & $345-346,668,673,729$ \\
\hline 21,24 & 380 & $2,5-10$ & $691-692,827$ \\
\hline 22,10 & 783 & 2,6 & 829 \\
\hline 23,2 & 784 & $2,6-10$ & 155 \\
\hline 23,15 & 381,409 & 2,9 & 405 \\
\hline 23,22 & 789 & 2,10 & 405 \\
\hline 23,31 & 786 & 2,12 & $827-828$ \\
\hline 24,11 & 432 & $2,12-13$ & 828 \\
\hline 24,23 & 786 & 2,13 & 679 \\
\hline 24,25 & 205 & 2,14 & 423 \\
\hline 25,11 & 350,361 & 2,16 & 415 \\
\hline 25,16 & 408 & 2,25 & $816-817$ \\
\hline 26 & 783 & $2,25-26$ & 817 \\
\hline 26,4 & 352 & 2,26 & 708 \\
\hline 26,5 & 351 & $2,26-27$ & $679-680$ \\
\hline 26,20 & 789 & 2,27 & 825 \\
\hline 26,29 & 655 & $3-4$ & 896 \\
\hline 27,13 & 740 & 3,2 & 405 \\
\hline 27,39 & 743 & 3,3 & $660,708-709$ \\
\hline $28,21-22$ & 209 & 3,4 & $420,680,685,760$ \\
\hline
\end{tabular}




\begin{tabular}{|c|c|c|c|}
\hline 3,5 & $647,710,716$ & 5,13 & 167 \\
\hline 3,6 & 716,760 & 5,14 & 167 \\
\hline 3,7 & 659 & 5,15 & $660,679,810$ \\
\hline 3,8 & $133-134,711-712,834$ & $5,15-19$ & $803-804$ \\
\hline $3,10-14$ & 904 & 5,17 & 569,810 \\
\hline 3,12 & 422 & 5,18 & 683 \\
\hline $3,15-20$ & 904 & 5,19 & 683,685 \\
\hline 3,19 & 688,830 & 5,20 & $167,373-374$ \\
\hline 3,20 & $687-688,692$ & 6,1 & $710-711,716,760,834$ \\
\hline 3,21 & 688 & 6 & $534,726,754,813,909,1007$ \\
\hline $3,22-23$ & $358-359$ & $6-8$ & $534,888-889,904-906$ \\
\hline $3,23-30$ & 679 & & 929,965 \\
\hline 3,24 & 679 & 6,2 & 711,760 \\
\hline 3,25 & 740 & $6,3-14$ & $804-807,1007$ \\
\hline 3,26 & 679 & 6,4 & $362,726,755$ \\
\hline 3,28 & 679 & $6,4-6$ & 359 \\
\hline 3,29 & 682,705 & 6,5 & $678-680,810$ \\
\hline 3,30 & $663,678-679,681,682,829$ & 6,6 & 693,755 \\
\hline 3,31 & 760 & 6,8 & $660,679-680,725-726$ \\
\hline 4,1 & 710,716 & 6,9 & 354,696 \\
\hline 4 & $687,906,965$ & 6,10 & $352-353,362,756$ \\
\hline $4-8$ & 965 & 6,13 & 628 \\
\hline 4,2 & 659,810 & $6,13-19$ & $752,753-757,1007$ \\
\hline 4,3 & 621 & 6,14 & 683 \\
\hline 4,5 & 679 & 6,15 & 712,760 \\
\hline $4,9-12$ & 980 & 6,17 & 205 \\
\hline 4,12 & $686-687$ & $6,17-18$ & 904 \\
\hline 4,13 & 700 & 6,20 & 415 \\
\hline 4,14 & 810 & 6,21 & 322 \\
\hline 4,18 & 686 & 6,22 & 158 \\
\hline 4,19 & 402 & 6,23 & 680,726 \\
\hline $4,20-21$ & $620-623,627$ & 7,1 & 355,430 \\
\hline 4,21 & 699 & 7 & 709 \\
\hline $4,23-24$ & $644-645$ & $7,1-8,11$ & $64,905-906$ \\
\hline 5,1 & 679 & $7,2-3$ & 817 \\
\hline 5 & 965 & 7,3 & 825 \\
\hline 5,2 & 295,734 & 7,5 & 416,816 \\
\hline 5,3 & 646,696 & 7,6 & 338 \\
\hline 5,6 & 431 & 7,7 & $316,322,665,710,716,753$ \\
\hline $5,6-9$ & 431 & & 760,810 \\
\hline 5,7 & $355,656,719-720$ & $7,7-23$ & 709 \\
\hline 5,8 & 682 & 7,9 & $407,444-445$ \\
\hline $5,8-10$ & $801-804$ & $7,9-10$ & $444-446$ \\
\hline 5,9 & $682-683,685$ & 7,10 & 389 \\
\hline 5,10 & 659,810 & 7,11 & $389,402,445$ \\
\hline 5,11 & 646 & 7,13 & $760-761$ \\
\hline 5,12 & 359 & $7,14-24$ & $318-319$ \\
\hline
\end{tabular}




\begin{tabular}{|c|c|c|c|}
\hline $7,16-20$ & 747 & 9,18 & 672,688 \\
\hline 7,17 & 639 & 9,19 & 490,714 \\
\hline 7,20 & 639 & $9,19-21$ & 672 \\
\hline 7,21 & 770 & 9,20 & 709 \\
\hline $7,22-23$ & 209 & $9,22-23$ & $667-673,770$ \\
\hline $7,24-25$ & 709 & $9,22-30$ & $659-660$ \\
\hline 7,25 & 205 & 9,24 & 671,687 \\
\hline 7,39 & 355 & $9,25-28$ & 687 \\
\hline 8,3 & $184-185$ & 9,27 & 231,690 \\
\hline $8,3-4$ & 46,63 & 9,29 & $665,687,700$ \\
\hline 8,9 & $658,662-663,810$ & 9,30 & $687-688,710,716$ \\
\hline $8,9-14$ & $806-808$ & $9,30-33$ & $205,689-690$ \\
\hline $8,12-17$ & 905 & $9,30-10,4$ & 864 \\
\hline 8,13 & 810,812 & 10,1 & 690 \\
\hline 8,14 & 355 & 10,2 & 690 \\
\hline 8,15 & $344,631-632,641$ & 10,3 & 282,764 \\
\hline $8,16-17$ & 663 & 10,6 & 757 \\
\hline 8,17 & 669 & $10,8-10$ & 818 \\
\hline 8,19 & 731 & $10,8-13$ & 690 \\
\hline $8,20-21$ & $731-732$ & 10,10 & 369 \\
\hline 8,22 & 347 & 10,11 & 690,692 \\
\hline 8,23 & $344,646,734$ & $10,11-15$ & $633-638,834$ \\
\hline 8,25 & 747 & 10,14 & 834 \\
\hline 8,28 & 696 & 10,15 & 971 \\
\hline $8,29-30$ & $241-242$ & 10,18 & $635-636,657-658$ \\
\hline 8,30 & $359,634,679,710,722$ & $10,18-21$ & 687 \\
\hline 8,31 & $710,716,812$ & 10,19 & 633 \\
\hline $8,31-32$ & $621,622,627,638,710$ & 11,1 & $760-761$ \\
\hline 8,32 & 711 & 11,11 & $373,669,760-761$ \\
\hline 8,33 & $679,709-710,722$ & 11,13 & 430,731 \\
\hline 8,34 & $343,647,652,710$ & 11,14 & 704,731 \\
\hline 8,35 & 710,722 & 11,15 & 810 \\
\hline 8,37 & 722 & 11,16 & 811 \\
\hline $8,38-39$ & $628,710,722-723$ & 11,17 & $811-812$ \\
\hline 9-11 & $155,668-669,947,980$ & $11,17-18$ & 660 \\
\hline 9,3 & 762,764 & $11,17-19$ & 490 \\
\hline $9,6-8$ & $688-689$ & $11,17-24$ & 390,987 \\
\hline $9,6-10,21$ & 64 & $11,18-23$ & 812 \\
\hline 9,9 & $686,700,716$ & 11,19 & 490,714 \\
\hline $9,9-10$ & 646 & $11,19-22$ & $819-820$ \\
\hline 9,11 & 309,628 & $11,19-24$ & $808-812,820$ \\
\hline $9,11-12$ & 402 & $11,20-21$ & 745 \\
\hline 9,12 & 686 & 11,21 & $659,750,810$ \\
\hline 9,14 & $710,716,760$ & 11,23 & 816 \\
\hline 9,15 & 688 & 11,24 & $390,659,810$ \\
\hline 9,16 & 672,688 & 11,25 & $411,766,831$ \\
\hline 9,17 & $671-672,737$ & 11,26 & $682-683,685,690,692$ \\
\hline
\end{tabular}




\begin{tabular}{|c|c|c|c|}
\hline 11,27 & 437 & 15,16 & 609 \\
\hline 11,30 & $407,445-446$ & $15,18-19$ & 604 \\
\hline 11,31 & 445 & $15,18-32$ & $602-613,970,1005$ \\
\hline 11,34 & 359 & 15,20 & 742 \\
\hline 11,36 & 49,848 & 15,21 & 687,971 \\
\hline 12,1 & $776-777$ & $15,21-22$ & $604-606,686$ \\
\hline $12,9-19$ & 753 & 15,22 & 369 \\
\hline 12,15 & 753 & $15,23-24$ & $606-607$ \\
\hline 12,16 & 736 & 15,24 & $430,447-448,733,742-743$ \\
\hline 12,18 & 234,642 & & $818-819,1007$ \\
\hline 12,19 & 751 & $15,24-25$ & 607 \\
\hline $12,19-20$ & $690-691$ & $15,25-26$ & 843 \\
\hline 12,20 & 917 & 15,26 & 352 \\
\hline 13,1 & 783,810 & $15,26-28$ & $607-608$ \\
\hline $13,1-4$ & $826-827$ & 15,27 & $352,610,659,810$ \\
\hline $13,1-7$ & $76-77$ & $15,28-29$ & 608 \\
\hline 13,2 & 745 & 15,29 & $716,742-743$ \\
\hline 13,3 & 824 & 15,30 & $608-609,776-777,843$ \\
\hline $13,3-4$ & $745,751,765-766$ & 15,31 & $843-844$ \\
\hline 13,5 & 645,701 & $15,31-32$ & $609-611$ \\
\hline 13,6 & 290,737 & 15,33 & 685 \\
\hline $13,8-10$ & 758 & 16,1 & 350 \\
\hline 13,9 & 753 & 16,2 & 830 \\
\hline 13,11 & 396 & $16,3-16$ & 753 \\
\hline $13,11-14$ & 757-759, 921-922 & 16,7 & 408 \\
\hline 13,12 & 757 & 16,17 & $368,685,776$ \\
\hline 13,13 & 757 & 16,19 & $343,685,770$ \\
\hline $14,1-5$ & $683-685$ & 16,20 & 685 \\
\hline 14,2 & 725 & 16,21 & 350 \\
\hline 14,4 & 761 & 16,22 & $154-155$ \\
\hline 14,8 & 917 & 16,23 & 350 \\
\hline $14,8-9$ & 846 & $16,25-26$ & 848 \\
\hline 14,9 & $351-353,389$ & 16,29 & 642 \\
\hline 14,10 & $684-685,853$ & & \\
\hline $14,10-12$ & 691 & \multicolumn{2}{|c|}{ Der erste Brief an die Korinther } \\
\hline 14,11 & $685,691-692,698,853$ & Brief & $437,727,898$ \\
\hline 14,12 & 685 & 1,1 & 4 \\
\hline 14,13 & 757 & 1,4 & $426-427$ \\
\hline 14,14 & 722 & 1,7 & 734 \\
\hline 14,15 & 810 & 1,8 & 829,921 \\
\hline 14,19 & 757 & 1,10 & 777 \\
\hline 14,21 & 628 & $1,10-15$ & 658 \\
\hline 14,23 & 826 & 1,11 & 434 \\
\hline 15,3 & $761-762$ & 1,14 & $648-649$ \\
\hline 15,5 & 736 & 1,16 & 650 \\
\hline $15,8-12$ & 689 & $1,18-30$ & 864 \\
\hline 15,13 & 420,761 & 1,21 & $352-353$ \\
\hline
\end{tabular}




\begin{tabular}{|c|c|c|c|}
\hline 2,2 & 810 & 7,6 & 647 \\
\hline $2,5-8$ & 667 & 7,7 & 770 \\
\hline 2,7 & 408,667 & 7,8 & 790 \\
\hline 2,8 & 370,810 & 7,10 & $639-640,647,789-790$ \\
\hline 2,11 & 810 & 7,12 & 790 \\
\hline 2,14 & 693 & 7,16 & 704 \\
\hline $2,14-15$ & 206 & 7,17 & 787 \\
\hline 2,16 & 359,829 & 7,22 & 237 \\
\hline 3,2 & 617 & 7,25 & 379 \\
\hline 3,4 & 419,423 & 7,26 & 728 \\
\hline 3,5 & 4 & 7,28 & $579,770-771$ \\
\hline 3,7 & 628 & 7,29 & 753 \\
\hline 3,10 & 813 & 7,32 & 771 \\
\hline $3,11-15$ & $812-813$ & 7,33 & 771 \\
\hline 3,13 & 434,707 & 7,35 & 830 \\
\hline $3,14-15$ & $801,814-815$ & 7,36 & 728 \\
\hline $3,16-17$ & 813 & 7,39 & 355,430 \\
\hline 3,18 & 727,832 & 7,40 & 727 \\
\hline $4,1-4$ & 684 & 8,1 & 239 \\
\hline 4,2 & 764 & 8,2 & 727 \\
\hline 4,5 & $408,410,429-430,438,829$ & 8,4 & 239 \\
\hline 4,7 & 659 & 8,5 & 663 \\
\hline 4,9 & $657,674,728-729$ & 8,6 & 49 \\
\hline 4,14 & 360,380 & 8,7 & 239 \\
\hline 4,16 & 776,829 & 8,8 & 628 \\
\hline $4,16-17$ & $839-840$ & 8,9 & 744 \\
\hline 4,17 & 829 & 8,10 & 404 \\
\hline 4,19 & 477,716 & $8,10-11$ & $239-240,523$ \\
\hline 5,1 & 360,483 & 8,11 & 920,922 \\
\hline 5,3 & 777 & 8,13 & $308,834-835$ \\
\hline $5,6-8$ & 916 & 8,14 & 640 \\
\hline 5,7 & 832 & 8,15 & 640 \\
\hline 5,8 & 628 & 9,10 & 734 \\
\hline $5,9-10$ & 664 & 9,11 & 659 \\
\hline 5,11 & 628 & 9,12 & $619,643-644,830$ \\
\hline 6,1 & 824 & $9,14-15$ & $787-788$ \\
\hline 6,2 & 801 & 9,15 & 360,716 \\
\hline $6,2-3$ & $694-695,814$ & 9,17 & 747,810 \\
\hline 6,5 & 716 & $9,17-18$ & 801 \\
\hline 6,9 & 705 & 9,19 & 232 \\
\hline $6,9-10$ & $628,694-695$ & $9,19-23$ & $844-845$ \\
\hline 6,11 & 679 & $9,20-21$ & 125 \\
\hline 6,12 & 716 & 9,21 & 480 \\
\hline 6,16 & 694 & 9,23 & 830 \\
\hline 6,18 & 830 & 9,24 & $233,697,832$ \\
\hline 7 & 870 & 9,27 & 750 \\
\hline 7,5 & 832 & 10,1 & 766,898 \\
\hline
\end{tabular}




\begin{tabular}{|c|c|c|c|}
\hline $10,1-22$ & 899,916 & 13,3 & $437,633,834$ \\
\hline 10 & $905-906$ & 13,10 & 437 \\
\hline 10,5 & $226,352-353$ & 13,11 & $323,396,417-418$ \\
\hline 10,6 & 899 & 14,5 & $449,649,670,716,765$ \\
\hline 10,7 & 389,903 & 14,10 & 205,655 \\
\hline $10,7-10$ & 628 & 14,12 & 282,764 \\
\hline 10,8 & $389,390-391,453$ & 14,13 & 780 \\
\hline 10,10 & $389-391,453$ & $14,14-15$ & 779 \\
\hline 10,11 & 899 & 14,16 & 707 \\
\hline 10,12 & 744 & 14,19 & 772 \\
\hline 10,13 & 829 & 14,25 & $788,852,899$ \\
\hline 10,20 & $766-767$ & 14,26 & 220,423 \\
\hline 10,21 & 766 & 14,34 & $797-798$ \\
\hline 10,27 & $768-769$ & 14,35 & 769 \\
\hline 10,28 & 769 & 14,37 & $360,727-728$ \\
\hline $10,32-33$ & 844 & 14,38 & 747 \\
\hline 11,2 & 790 & 15,1 & 494 \\
\hline 11,3 & 766 & $15,1-2$ & 389 \\
\hline $11,4-5$ & 779 & 15 & 62,456 \\
\hline 11,6 & 810 & $15-16$ & 439,453 \\
\hline 11,7 & 796 & 15,2 & $389,650,661-662,810$ \\
\hline 11,11 & 628,650 & $15,2-3$ & 485 \\
\hline 11,13 & 331,779 & $15,3-5$ & 7 \\
\hline 11,17 & $789-791$ & 15,4 & $312,354,368$ \\
\hline $11,17-32$ & $665-667$ & $15,5-7$ & 393 \\
\hline 11,18 & $25,205,360,405$ & 15,10 & $621-622,627,638-640,647$ \\
\hline 11,19 & 701 & 15,12 & 362 \\
\hline $11,20-22$ & 405 & $15,13-14$ & 54,634 \\
\hline 11,21 & 420 & $15,14-16$ & $809-810$ \\
\hline 11,22 & 653,833 & 15,15 & 663 \\
\hline 11,23 & 369,392 & 15,16 & 810 \\
\hline $11,23-33$ & $790-792$ & 15,17 & 431 \\
\hline 11,24 & $309,391-392,402,453$ & 15,19 & 361 \\
\hline 11,25 & $301,392,402,423-424,454$ & $15,22-28$ & 524 \\
\hline 11,26 & $344,410,423-424,430,899$ & $15,23-24$ & 393 \\
\hline 11,27 & 830 & 15,24 & $393,418-419,423,436-438$ \\
\hline 11,28 & 405 & 15,25 & $410-411,701$ \\
\hline 11,29 & $821,824-826$ & 15,26 & 411 \\
\hline 11,31 & $617,665-666,821$ & 15,27 & 438,699 \\
\hline 11,32 & 831 & 15,28 & $395,438,832$ \\
\hline 11,33 & 720,833 & 15,31 & 343,689 \\
\hline 11,34 & $416,430,787,832-833,853$ & 15,32 & 659 \\
\hline 12,1 & 766 & 15,34 & 756 \\
\hline 12,2 & $125,369,416,424,766$ & 15,35 & 714,717 \\
\hline 12,11 & 647,774 & 15,37 & 656 \\
\hline 12,28 & 205,393 & 15,38 & 235 \\
\hline 13,2 & 372,725 & 15,41 & 205 \\
\hline
\end{tabular}




\begin{tabular}{|c|c|c|c|}
\hline 15,45 & 393 & $1,23-2,22$ & 715 \\
\hline 15,46 & $393,405-406$ & 2,1 & 748 \\
\hline 15,49 & 359,579 & 2,2 & 810 \\
\hline 15,52 & 701 & 2,3 & $25,664,737-738$ \\
\hline 15,53 & 701 & 2,4 & 25,693 \\
\hline 15,54 & $395,438,768$ & 2,6 & 466 \\
\hline 15,57 & 205 & 2,7 & 750 \\
\hline 15,58 & $426,696-697$ & 2,8 & $776-777$ \\
\hline 16,1 & 787 & 2,9 & 25,693 \\
\hline \multirow[t]{2}{*}{16,2} & $395,438-439,787,830$ & 2,10 & 810 \\
\hline & 832,834 & 2,12 & 368,930 \\
\hline $16,2-3$ & $430,438-439$ & $2,12-13$ & $3,632,930,932,935,967$ \\
\hline $16,2-8$ & $718-719$ & 2,13 & $295-296,319,322,366-367$, \\
\hline 16,3 & $26,395,439,453$ & & $387,402,732-733,931,935$, \\
\hline 16,5 & $394-395,418,439,454,716$ & & 965 \\
\hline $16,5-8$ & $715-716$ & $2,13-14$ & 930-932, 935 \\
\hline 16,6 & 832 & 2,14 & $87,205-206,366,398,426-427$, \\
\hline $16,6-7$ & 767 & & $451,927,930-936,1009$ \\
\hline 16,7 & $472,597,733$ & $2,14-16$ & 133 \\
\hline 16,9 & 291,295 & $2,14-7,4$ & 930 \\
\hline 16,10 & 744,763 & $2,15-16$ & $933-934$ \\
\hline 16,11 & 720 & 2,16 & 934 \\
\hline 16,12 & 439,777 & 3,1 & 26 \\
\hline \multirow[t]{2}{*}{16,15} & 783 & 3 & 934 \\
\hline & & 3,2 & 360 \\
\hline \multicolumn{2}{|c|}{ Der zweite Brief an die Korinther } & $3,7-8$ & 660 \\
\hline \multirow[t]{2}{*}{ Brief } & $322,466,445,748-750$ & 3,9 & 779,810 \\
\hline & 767-768, 932 & 3,11 & 810 \\
\hline 1,1 & 4 & $3,15-16$ & 424 \\
\hline $1-7$ & 932 & 3,18 & 343,345 \\
\hline $1-9$ & 466 & 4,2 & 628 \\
\hline 1,6 & 360 & 4,3 & 363 \\
\hline 1,7 & 360,696 & $4,3-6$ & 908 \\
\hline 1,8 & $377,489,766$ & 4,6 & 908 \\
\hline $1,8-9$ & $122-123,367,935$ & 4,7 & 427 \\
\hline 1,9 & $295,366-367,732,777$ & $4,7-10$ & 450 \\
\hline 1,10 & $295,361,367,732$ & 4,8 & 380 \\
\hline 1,11 & 369,501 & 4,10 & $359,427,450$ \\
\hline 1,13 & 154,736 & 4,11 & 830 \\
\hline 1,15 & 774 & 4,18 & 380 \\
\hline $1,15-16$ & $391,403-404,715$ & 5,1 & $696-697$ \\
\hline $1,15-17$ & 743 & $5,1-5$ & $767-768$ \\
\hline 1,16 & 930 & 5,2 & 344,738 \\
\hline 1,17 & $715,774,930-931$ & $5,2-3$ & 661 \\
\hline 1,18 & 698 & 5,4 & 831 \\
\hline $1,18-22$ & $715-716$ & 5,5 & 737 \\
\hline 1,23 & 698 & 5,8 & 764 \\
\hline
\end{tabular}




\begin{tabular}{|c|c|c|c|}
\hline 5,11 & 345,732 & 9,15 & 205 \\
\hline 5,12 & 830 & $10-13$ & $313,466,716$ \\
\hline $5,15-17$ & 846 & 10,2 & 747 \\
\hline 5,17 & 205,659 & $10,2-3$ & 233 \\
\hline 5,20 & 379 & 10,3 & 231 \\
\hline 5,21 & 126 & $10,3-6$ & 233 \\
\hline 6,1 & 777 & 10,5 & 732 \\
\hline 6,2 & 205 & 10,6 & 439 \\
\hline 6,9 & 205 & 10,13 & 716 \\
\hline 6,11 & 291, 295, 360 & 11,1 & 675 \\
\hline 6,12 & 360 & $11,1-4$ & $348,746-747$ \\
\hline $6,14-7,1$ & 905 & 11,2 & 835,921 \\
\hline 6,16 & 309,320 & 11,3 & 835 \\
\hline 7,3 & 360,700 & 11,4 & 810 \\
\hline 7,4 & 935 & 11,5 & 746 \\
\hline 7,5 & $366-368,376,930,965$ & 11,6 & 344 \\
\hline $7,5-7$ & 3,930 & 11,8 & 402 \\
\hline 7,6 & $366-367$ & 11,9 & 619,717 \\
\hline $7,6-7$ & $466,645-647$ & 11,10 & 698 \\
\hline 7,8 & $641,659,810$ & 11,11 & 190 \\
\hline 7,9 & 383,641 & 11,12 & 832 \\
\hline $7,9-12$ & 749 & $11,13-15$ & 747 \\
\hline 7,11 & 205,737 & 11,15 & 829 \\
\hline 7,12 & 659 & 11,16 & $717,781,823,832,834$ \\
\hline 7,14 & $660-661$ & 11,18 & 716 \\
\hline 8,1 & 494 & 11,19 & 717 \\
\hline 8 & 313 & 11,20 & 747,810 \\
\hline 8,4 & 642 & 11,21 & $296,374,830$ \\
\hline 8,5 & $405,641-642$ & 11,25 & $319-320,330-331,365$ \\
\hline 8,6 & 313,777 & 11,28 & 744 \\
\hline 8,7 & 753 & 11,30 & 822 \\
\hline 8,10 & 644 & $11,32-33$ & 485 \\
\hline 8,11 & 741 & 11,33 & 453 \\
\hline 8,12 & 741,810 & 12,1 & $485-487,701,716-717$ \\
\hline 8,16 & 205 & $12,1-6$ & $391,511-515,653,820-824$, \\
\hline 8,18 & 313 & & 969,1002 \\
\hline 8,19 & 646,741 & 12 & $120,133,562$ \\
\hline 8,21 & 645 & 12,2 & $378,408,653,657$ \\
\hline 8,22 & 313,327 & $12,2-4$ & 464,487 \\
\hline 9,2 & 360,741 & $12,3-4$ & 378 \\
\hline 9,3 & 360,777 & 12,4 & 453 \\
\hline $9,3-5$ & 839 & 12,5 & 716 \\
\hline 9,4 & 654 & 12,6 & $487,653-654,769-770$ \\
\hline 9,6 & 420 & & $820-824,1006$ \\
\hline 9,8 & 426,761 & 12,7 & 464 \\
\hline $9,11-13$ & 739 & 12,8 & $331,366,779$ \\
\hline 9,14 & 739 & $12,8-9$ & 320,467 \\
\hline
\end{tabular}




\begin{tabular}{|c|c|c|c|}
\hline 12,9 & $290,320-321,366,716$ & 1,11 & $258,381,399,492,494-495$ \\
\hline 12,10 & $366,423,764,822$ & $1,11-12$ & $494-495,509,575$ \\
\hline 12,11 & 664,810 & $1,11-17$ & 340 \\
\hline 12,13 & 649,810 & 1,12 & $363,399,492,495-497,499$ \\
\hline 12,14 & $205,391,796-797$ & & $504,506,508,628$ \\
\hline $12,14-15$ & 717 & $1,12-2,14$ & 16,871 \\
\hline 12,15 & $704-705$ & 1,13 & $236,281,301,407,443,487$ \\
\hline $12,17-18$ & $312-313,366-367,466$ & & $488,492,495-496,501,521$, \\
\hline 12,19 & 343,347 & & 596,599 \\
\hline $12,20-21$ & $748-750$ & $1,13-14$ & 492, 494, 496-499, 575, 970 \\
\hline 12,21 & 617,700 & $1,13-17$ & $130-131,233-234,249-260$ \\
\hline 13,1 & 288 & & $492,497-500,1013$ \\
\hline 13,2 & $414,700,715,749,822$ & $1,13-24$ & $255,257,260$ \\
\hline 13,4 & $352-353$ & $1,13-2,10$ & 507,510 \\
\hline 13,5 & $650,661-662,801$ & $1,13-2,21$ & $7,9,128,255,391,453,455$, \\
\hline 13,6 & 693,733 & & $490,492,510-511,515,970$ \\
\hline 13,7 & 764 & & $1000-1001,1013$ \\
\hline 13,9 & $423,764-765$ & 1,14 & $236,979,980$ \\
\hline 13,10 & $25,360,830$ & 1,15 & $352,396,398-399,434-435$ \\
\hline 13,11 & 736 & & $593,638,899$ \\
\hline & $1,15-16$ & $438,496-497,575$ \\
\hline \multicolumn{2}{|c|}{ Der Brief an die Galater } & $1,15-17$ & $168,445,468-470,492$, \\
\hline Brief & $52-53,155-156,511,556$ & & 498,1013 \\
\hline & $849-850,895,947-949$ & $1,15-21$ & 469 \\
\hline 1,1 & 4, 495, 575 & 1,16 & $123,399,435,473,575-576$ \\
\hline $1-2$ & $64,146,453,456,524,947$ & & $593,638-639,861,1013$ \\
\hline $1-4$ & 479 & $1,16-17$ & $246-249,398-399,497,575$, \\
\hline 1,3 & $841-842,985$ & & 628,1013 \\
\hline 1,4 & $\begin{array}{l}477,524,653,830,840-850 \\
970,984-985,988,1006-1007 \\
1011,1014\end{array}$ & 1,17 & $\begin{array}{l}254,340,391,398,408,453, \\
468,497-499,506,564,648, \\
979,1013\end{array}$ \\
\hline 1,5 & $848-849$ & $1,17-18$ & $216-217$ \\
\hline 1,6 & $\begin{array}{l}476-478,487-488,497,510, \\
520-522,562-563,566,599, \\
774,849,1014\end{array}$ & 1,18 & $\begin{array}{l}255,261,308,391,393,401, \\
491,500,505,592,597-598, \\
830,835,854,1013\end{array}$ \\
\hline $1,6-7$ & $493,510,650-651,773$ & $1,18-19$ & $472,499,638,1013$ \\
\hline $1,6-9$ & 495 & $1,18-20$ & 498,506 \\
\hline 1,7 & $478-479,511,649,747,773-774$ & $1,18-21$ & 520,1013 \\
\hline 1,8 & $231,381,515,774$ & 1,19 & 308,648 \\
\hline $1,8-9$ & $493,495,508-509,511$ & $1,19-21$ & 236 \\
\hline $1,8-10$ & 510 & 1,20 & $205,255,360,499,502,595$ \\
\hline 1,9 & $349,360,515,700$ & & $623,698,912,1002$ \\
\hline $1,9-12$ & 253 & 1,21 & $107,255,261,288,393,469$, \\
\hline \multirow[t]{2}{*}{1,10} & $282,485,492-494,503,509$ & & $491,499-500,1013$ \\
\hline & $560,665,667,764,821$ & $1,21-22$ & 208 \\
\hline $1,10-11$ & 1013 & $1,21-24$ & 506 \\
\hline $1,10-2,21$ & $65,492-511,1013$ & $1,21-2,1$ & $464-465,1013$ \\
\hline
\end{tabular}




\begin{tabular}{|c|c|c|c|}
\hline $1,22-24$ & $246,255,499$ & $3,1-5$ & $509,515,517$ \\
\hline \multirow[t]{2}{*}{1,23} & $235-236,331,407,442-443$ & $3,1-6$ & 490,515 \\
\hline & $469,500,518,523,970,1013$ & $3,1-4,11$ & $866-867,874-876,904,919$ \\
\hline 1,24 & 500 & $3,1-4,31$ & 875 \\
\hline \multirow[t]{2}{*}{2,1} & $255,261,308,391,393,453$ & 3 & 455 \\
\hline & $491,500,1013$ & $3-4$ & $63,515,896,904$ \\
\hline $2,1-2$ & 209 & 3,2 & 640,766 \\
\hline $2,1-10$ & $7,498,500-504,507$ & $3,2-5$ & 462 \\
\hline \multirow[t]{3}{*}{2,2} & $122,469,498,563-564$ & 3,3 & 562,599 \\
\hline & $594-600,617,654-655,724$ & 3,4 & 564,661 \\
\hline & 750,1013 & 3,5 & $462,479,599,924$ \\
\hline 2,3 & $231,511,598-599$ & $3,5-6$ & 237 \\
\hline 2,4 & $123,364,851,1013$ & 3,6 & 924 \\
\hline $2,4-5$ & $498,594-601$ & 3,7 & $462,515-516,693$ \\
\hline 2,5 & $364,498,510,830,835,1013$ & 3,8 & $604,679,700,896,948$ \\
\hline \multirow[t]{2}{*}{2,6} & $208,509,594-595,598,600$ & 3,10 & 374 \\
\hline & 639,707 & 3,11 & $346,698-699,919-920$ \\
\hline $2,6-9$ & 507 & 3,13 & 462 \\
\hline $2,6-10$ & $234-235,598-599,642$ & $3,13-14$ & $62-63,123-126,867,917$ \\
\hline 2,7 & 121,469 & & $951-952,968$ \\
\hline 2,9 & $121,507,639$ & 3,14 & 557 \\
\hline 2,10 & $359,500-504,507,737,753$ & $3,15-17$ & 980 \\
\hline $2,10-11$ & 209 & 3,16 & $920,923,948$ \\
\hline \multirow[t]{2}{*}{2,11} & $128,205,396-397,454$ & 3,17 & $401-402$ \\
\hline & $480-481,1013$ & $3,17-25$ & 923 \\
\hline $2,11-21$ & $498,504-511,596,745$ & 3,18 & $695,810,923$ \\
\hline \multirow[t]{2}{*}{2,12} & $364,382,396-397,409$ & 3,19 & $373-374,410-411,455$ \\
\hline & $454-455,658,745,1013$ & & $786-787$ \\
\hline $2,12-13$ & 346,560 & 3,21 & $665,667,810,852$ \\
\hline 2,13 & $346,372,377$ & 3,22 & 462,948 \\
\hline \multirow[t]{2}{*}{2,14} & $168,205,319,346,396-398$ & 3,23 & $236,323,409,413,455,948$ \\
\hline & $454,480-481,560,658$ & $3,23-25$ & $461-463,478-479,524,923$, \\
\hline $2,15-17$ & 124 & & 927,1001 \\
\hline $2,15-21$ & 65,364 & 3,24 & $323-324,413-414,844$ \\
\hline \multirow[t]{2}{*}{2,16} & $209,346,352,649,679$ & 3,25 & $324,402,948$ \\
\hline & $687-688,696$ & 3,26 & $479,558,921,923,926$ \\
\hline $2,16-17$ & $593-594,601,1013$ & $3,26-29$ & $516,921-923$ \\
\hline 2,17 & $282,259,704,764,853$ & 3,27 & $634,921,923$ \\
\hline 2,18 & 810 & 3,28 & $479,565,921,923,948,1002$ \\
\hline 2,19 & 480,693 & 3,29 & $479,558,634,920,922-923$ \\
\hline $2,19-20$ & 846 & & $925-926$ \\
\hline 2,20 & 362,639 & 4,1 & $221,355,430$ \\
\hline $2,20-21$ & 617 & $4,1-2$ & $923-927,963,966$ \\
\hline 2,21 & $477,490,660,810,849$ & $4,1-3$ & 557 \\
\hline \multirow[t]{2}{*}{3,1} & $462,507,509-510,903,920$ & $4,1-6$ & 411,561 \\
\hline & 923,1013 & $4,1-7$ & 923-927, 1014 \\
\hline $3,1-4$ & 599 & $4,1-11$ & 566 \\
\hline
\end{tabular}




\begin{tabular}{|c|c|c|c|}
\hline \multirow[t]{4}{*}{$4,1-12$} & $557-567,568,652-653$ & $4,21-29$ & 481 \\
\hline & $849-850,875,920,938,952$, & 4,25 & 979 \\
\hline & $963,966,1004-1005,1007$ & 4,30 & $479-481,567,1013$ \\
\hline & 1010 & 4,31 & 752 \\
\hline 4 & 937 & 5,2 & $309,674-675$ \\
\hline 4,2 & 411, 430, 929 & 5,4 & 346 \\
\hline $4,2-6$ & 563 & 5,5 & 734 \\
\hline $4,2-10$ & 563 & 5,7 & 326,736 \\
\hline 4,3 & $316,415,558,847,966$ & 5,8 & 476,736 \\
\hline \multirow[t]{4}{*}{$4,3-6$} & $62-63,115,123-126,490$ & 5,10 & $735-738,830$ \\
\hline & $561-562,564-566,652-653$ & 5,13 & 476,479 \\
\hline & $867,917,920-921,923-927$ & 5,16 & 372 \\
\hline & $952,963,966,968,1004-1005$ & 5,17 & $373-374,830$ \\
\hline $4,3-11$ & 563,565 & 5,18 & 747 \\
\hline 4,4 & 398,557 & 5,20 & 748 \\
\hline $4,4-6$ & $557-558$ & 5,21 & 309,700 \\
\hline 4,6 & 966 & 6,1 & 231,659 \\
\hline 4,7 & $558,561-563,565-566,966$ & 6,3 & 728,810 \\
\hline $4,7-10$ & 563 & 6,4 & 639 \\
\hline 4,8 & 966 & 6,7 & 830 \\
\hline $4,8-9$ & $115,490,558-562,968$ & 6,9 & 430,825 \\
\hline $4,8-10$ & $562-564,963,1004-1005$ & 6,10 & 430 \\
\hline $4,8-11$ & $564-566$ & 6,11 & 154,349 \\
\hline $4,8-12$ & $952,1004-1005$ & 6,12 & $346-347,481,745,771$ \\
\hline \multirow[t]{2}{*}{4,9} & $479,564,566-567,622,647$ & $6,12-13$ & 478,773 \\
\hline & $652,771,847,849-850,969$ & 6,13 & 347 \\
\hline $4,9-11$ & 969 & $6,13-14$ & 840 \\
\hline 4,10 & $562-563,771$ & 6,14 & 511,649 \\
\hline $4,11-12$ & $920-922,963,1004-1005$ & 6,15 & 628 \\
\hline 4,11 & $\begin{array}{l}302,521,563-566,564,566 \\
568,617,652,654,745-746\end{array}$ & 6,16 & 829,948 \\
\hline \multirow[t]{3}{*}{4,12} & $479-481,490,511,515-516,564$ & \multicolumn{2}{|c|}{ Der Brief an die Epheser } \\
\hline & $566-568,579,622,751,754$ & Brief & 26,125 \\
\hline & 1001-1002, 1013 & 1,1 & 4 \\
\hline $4,12-16$ & 404 & 1,4 & 409,455 \\
\hline $4,12-19$ & 65,947 & 1,9 & 740 \\
\hline 4,13 & 40,665 & 1,14 & 695 \\
\hline $4,13-14$ & $480,564,567$ & 1,15 & 779 \\
\hline 4,14 & $622,627-628$ & 1,16 & 427 \\
\hline 4,15 & $567,659,665$ & 1,17 & 779 \\
\hline $4,15-16$ & 947 & 1,23 & 779 \\
\hline 4,16 & 567 & $2,1-5$ & 444,447 \\
\hline 4,17 & $478,567,639,771-772$ & $2,2-3$ & 417 \\
\hline 4,18 & $420-421,426$ & 2,6 & 389 \\
\hline 4,19 & $411-413,564-565$ & 2,8 & 363 \\
\hline 4,20 & 567,674 & 2,10 & 830 \\
\hline 4,21 & $479,567,771$ & 2,11 & 488 \\
\hline
\end{tabular}




\begin{tabular}{|c|c|c|c|}
\hline $2,11-13$ & $441-443,523$ & 1,7 & 236 \\
\hline 2,12 & 445 & 1,8 & 739 \\
\hline 2,13 & 354,407 & 1,9 & $344,779-780$ \\
\hline 3,1 & 4,661 & 1,10 & 414 \\
\hline 3,2 & 661 & 1,12 & 295,774 \\
\hline 3,14 & 779 & $1,12-13$ & 377 \\
\hline 3,16 & 779 & 1,16 & 696,701 \\
\hline 3,21 & 848 & 1,17 & 288 \\
\hline 3,29 & 848 & 1,20 & $426,731-732$ \\
\hline 4,1 & 776 & $1,21-26$ & 737 \\
\hline $4,10-14$ & 412 & 1,22 & 707 \\
\hline 4,13 & $412-413$ & 1,23 & 739 \\
\hline $4,13-16$ & 412 & $1,24-25$ & 735 \\
\hline $4,17-21$ & 661 & 1,25 & 694 \\
\hline 4,18 & 363 & 1,27 & 343,832 \\
\hline 4,21 & 488 & 1,29 & 644 \\
\hline 4,25 & 227,833 & 1,30 & 236,644 \\
\hline 4,26 & 756 & 2,2 & $735-736$ \\
\hline 4,27 & 628 & 2,3 & 628 \\
\hline 4,28 & 652,832 & $2,6-7$ & $622-623,627$ \\
\hline 4,29 & 832 & $2,6-11$ & $45,62,64$ \\
\hline 4,30 & 414 & $2,7-8$ & 622,850 \\
\hline 5,2 & 237 & 2,8 & 413 \\
\hline 5,3 & 628 & 2,9 & $389,850,854$ \\
\hline 5,5 & $695-697,753$ & $2,9-11$ & 920 \\
\hline 5,11 & 652 & $2,9-10$ & $125-126,850$ \\
\hline 5,20 & 426 & 2,10 & 622,698 \\
\hline 5,33 & 744,753 & $2,10-11$ & $850-854$ \\
\hline 6,3 & 853 & 2,11 & 698 \\
\hline 6,4 & 438 & 2,12 & 426,741 \\
\hline 6,13 & 832 & 2,13 & 644 \\
\hline 6,14 & 238 & $2,14-16$ & 580 \\
\hline 6,16 & 829 & 2,16 & $326,414,580,628$ \\
\hline 6,17 & 780 & 2,19 & $733,737-738$ \\
\hline 6,19 & 780 & 2,22 & 693 \\
\hline 6,21 & 853 & 2,23 & $430,733,737-738$ \\
\hline $6,21-22$ & $707,837-839,1001$ & 2,24 & $716,737-738$ \\
\hline \multirow[t]{2}{*}{6,22} & $580,737,830$ & 2,25 & 348,739 \\
\hline & & 2,26 & $331,348,739$ \\
\hline \multicolumn{2}{|c|}{ Der Brief an die Philipper } & 2,28 & $126,580,739,837$ \\
\hline Brief & 16,466 & 2,30 & 413 \\
\hline 1,1 & 4 & $3,1-2$ & 466 \\
\hline 1,3 & 420,736 & $3,1-11$ & 884 \\
\hline $1,3-4$ & 426 & $3,2-11$ & 64 \\
\hline 1,4 & 451 & 3,6 & 123 \\
\hline 1,5 & $434,736-737$ & $3,7-8$ & 348 \\
\hline 1,6 & $736-738$ & 3,8 & 830 \\
\hline
\end{tabular}




\begin{tabular}{|c|c|c|c|}
\hline $3,8-12$ & $693-694$ & 2,12 & $725-726$ \\
\hline 3,11 & 694,731 & 2,14 & 296,348 \\
\hline $3,13-15$ & 736 & 2,20 & 659 \\
\hline 3,16 & 753 & 2,21 & 628 \\
\hline 3,18 & $327,330-331$ & 3,1 & 660 \\
\hline $3,19-20$ & $734-735$ & 3,4 & 395,439 \\
\hline 3,21 & 829 & $3,5-7$ & $416-417$ \\
\hline 4,2 & 736,776 & 3,7 & $416-417,425,430-431$ \\
\hline $4,2-3$ & 778 & $3,7-8$ & $407,446-447$ \\
\hline 4,4 & 426 & 3,8 & 447 \\
\hline 4,10 & 407 & 3,21 & 832 \\
\hline 4,11 & 289 & 3,22 & 744 \\
\hline 4,12 & 698 & 3,23 & 830 \\
\hline 4,14 & 650 & 3,24 & 694 \\
\hline 4,15 & $399-401$ & 3,10 & 414 \\
\hline 4,16 & $400-401$ & 3,17 & 830 \\
\hline 4,18 & 361 & 3,24 & 343 \\
\hline 4,19 & 848 & 4,2 & 421,780 \\
\hline \multirow[t]{2}{*}{4,20} & 848 & 4,3 & 421,611 \\
\hline & & 4,6 & 426 \\
\hline \multicolumn{2}{|c|}{ Der Brief an die Kolosser } & $4,7-9$ & $837-838,1014$ \\
\hline \multirow[t]{2}{*}{ Brief } & $133,156,470-471,726$, & 4,8 & $431,580,737,830$ \\
\hline & 1007,1014 & 4,9 & 762 \\
\hline 1,1 & 4 & 4,10 & 427 \\
\hline 1,2 & 432 & 4,12 & $344,426,450-451$ \\
\hline 1,3 & 344,427 & 4,16 & $133,439,839$ \\
\hline $1,4-9$ & $432-434$ & 4,17 & 126,830 \\
\hline 1,5 & 432 & & \\
\hline $1,5-8$ & 470 & \multicolumn{2}{|c|}{ Der erste Brief an die Thessalonicher } \\
\hline 1,6 & $222,432-433$ & 1,1 & 761 \\
\hline $1,7-8$ & 432,779 & 1,2 & 420,427 \\
\hline 1,8 & 434 & 1,5 & $645-646,707$ \\
\hline 1,9 & $222,432-433,779-780$ & 1,6 & 232,402 \\
\hline 1,13 & 442,843 & 1,8 & 645 \\
\hline 1,14 & 442 & 1,9 & 707,788 \\
\hline 1,17 & 408 & 1,10 & 354,723 \\
\hline 1,18 & 830 & $2,1-3,10$ & 16 \\
\hline 1,19 & 352 & $2,5-6$ & 628 \\
\hline $1,21-22$ & $442-443,446,470,1001$ & $2,5-7$ & 232,642 \\
\hline 1,22 & 921 & $2,5-9$ & $623-625,627,642$ \\
\hline 1,23 & 4,661 & 2,8 & $295,352,764$ \\
\hline $1,26-27$ & 766 & 2,9 & 488 \\
\hline 1,27 & 734,764 & 2,16 & $426,668-669,844$ \\
\hline 2,1 & 766 & 2,17 & $472,739-740$ \\
\hline 2,4 & 830 & 2,18 & 773 \\
\hline 2,5 & 810 & 2,19 & $705-706$ \\
\hline 2,8 & 744 & 3,1 & 352 \\
\hline
\end{tabular}




\begin{tabular}{|c|c|}
\hline $3,2-3$ & $700-701$ \\
\hline 3,3 & 696,701 \\
\hline 3,4 & $417,700-701$ \\
\hline 3,5 & $563,654-655,750$ \\
\hline 3,6 & $426,472,738-739$ \\
\hline 3,8 & 793 \\
\hline $3,9-11$ & $761-762$ \\
\hline 3,10 & 472 \\
\hline 3,14 & $219-220$ \\
\hline 4,1 & $205,343,707,778$ \\
\hline $4,3-4$ & 698 \\
\hline 4,6 & 700 \\
\hline 4,10 & $343,776,778-779$ \\
\hline $4,10-12$ & $789-790$ \\
\hline 4,11 & 793 \\
\hline 4,13 & $487-489,766$ \\
\hline $4,13-17$ & 845 \\
\hline 4,14 & $389,800,810$ \\
\hline $4,14-18$ & $488-489$ \\
\hline 4,15 & 349,414 \\
\hline $4,16-17$ & 406 \\
\hline 4,17 & $\begin{array}{l}393,421,426,455,524, \\
845-846\end{array}$ \\
\hline 5,2 & 696,735 \\
\hline 5,3 & $419,423,436$ \\
\hline $5,8-9$ & $731-732,734$ \\
\hline 5,9 & 845 \\
\hline 5,10 & $421,845-846$ \\
\hline 5,11 & 343 \\
\hline 5,12 & 778 \\
\hline 5,14 & 776 \\
\hline 5,15 & 426 \\
\hline 5,16 & 426 \\
\hline 5,23 & 723,921 \\
\hline 5,24 & 829 \\
\hline 5,25 & 611 \\
\hline
\end{tabular}

$\begin{array}{ll}2,3 & 447-449 \\ 2,5 & 431,488 \\ 2,7 & 410 \\ 2,8 & 392,438,454,524,829 \\ 2,8-10 & 632 \\ 2,13 & 426,726 \\ 3,1 & 25,611,780 \\ 3,1-2 & 670 \\ 3 & 937,963 \\ 3,2-4 & 793-794 \\ 3,3 & 738,792,829 \\ 3,4 & 736,738,792-793, \\ & 937,964 \\ 3,5-6 & 792-793 \\ 3,7-8 & 576,580-583,601,627-631, \\ & 769,937,963,970 \\ 3,7-12 & 696 \\ 3,9 & 417 \\ 3,10 & 417,628,769,793 \\ 3,12 & 417,777,793,937,964 \\ 3,14 & 832\end{array}$

Der erste Brief an Timotheus

Brief 26, 794, 824

$1,1 \quad 4$

$1,3 \quad 794-795$

$1,4 \quad 628$

$1,5 \quad 794$

$1,7 \quad 628,707,770$

1,12-13 404-405

1,12-14 485-487

$1,16 \quad 762$

1,16-17 848

$1,18 \quad 762,830$

$1,20 \quad 830$

$2,1 \quad 405,776$

2,1-3,13 733

$2,2 \quad 793$

Der zweite Brief an die Thessalonicher

2,3-4 772

$2,8 \quad 775$

2,9-10 775

2,11 797-798

2,12 797-798

$2,13 \quad 393$

$3,2 \quad 700$

3,5 697-698, 725

$3,7 \quad 701,762$ 


\begin{tabular}{|c|c|}
\hline $3,7-8$ & 238 \\
\hline 3,10 & $393,406,524$ \\
\hline 3,11 & 360 \\
\hline 3,14 & 360 \\
\hline $3,14-15$ & $733-734$ \\
\hline 4,1 & 309 \\
\hline $4,1-11$ & 795 \\
\hline $4,3-5$ & 795 \\
\hline 4,6 & $44,296,795,824$ \\
\hline 4,7 & 795 \\
\hline 4,10 & 295,732 \\
\hline 4,11 & 795 \\
\hline 4,13 & $429-430$ \\
\hline 4,15 & 832 \\
\hline 4,16 & 824 \\
\hline 5,3 & 795 \\
\hline 5,4 & 405,795 \\
\hline 5,5 & $295,361,795$ \\
\hline $5,5-6$ & 795 \\
\hline 5,7 & 795,832 \\
\hline $5,9-10$ & 660 \\
\hline $5,9-13$ & 421 \\
\hline 5,11 & $439-440,646,772-773$ \\
\hline 5,13 & $289,421,646-647$ \\
\hline 5,14 & 775 \\
\hline 5,16 & 832 \\
\hline 5,20 & 832 \\
\hline $5,21-22$ & 781 \\
\hline 5,22 & 628 \\
\hline 6,1 & 832,834 \\
\hline $6,3-4$ & 747 \\
\hline 6,5 & $728-729$ \\
\hline $6,6-11$ & $774-775$ \\
\hline 6,8 & 713,824 \\
\hline $6,11-12$ & 795 \\
\hline 6,12 & 818 \\
\hline 6,13 & $413,420,794-795$ \\
\hline 6,14 & $413,723,794$ \\
\hline 6,15 & 829 \\
\hline $6,17-19$ & 628,796 \\
\hline 6,20 & 723 \\
\hline \multicolumn{2}{|c|}{ Der zweite Brief an Timotheus } \\
\hline Brief & 26,156 \\
\hline 1,1 & 4 \\
\hline 1,3 & $423-424,427$ \\
\hline 1,4 & 472,739 \\
\hline
\end{tabular}

$\begin{array}{ll}1,5 & 447-448 \\ 1,8 & 628 \\ 1,9 & 408 \\ 1,10 & 724 \\ 1,12 & 361,414,722-724 \\ 1,13-14 & 723 \\ 1,14 & 724 \\ 1,16 & 327,331,762 \\ 1,16-17 & 625-627 \\ 1,17 & 327,390 \\ 1,18 & 325-326,723 \\ 2,2 & 723,829 \\ 2,6 & 405 \\ 2,9 & 413 \\ 2,10 & 830-831 \\ 2,11 & 659,810 \\ 2,11-13 & 814-816 \\ 2,12 & 801 \\ 2,19 & 359 \\ 2,24 & 796 \\ 2,25 & 720,750-751 \\ 3,1 & 693 \\ 3,7 & 427,451 \\ 3,8 & 237 \\ 3,11 & 707 \\ 3,12 & 771,777 \\ 3,15 & 434 \\ 4,2 & 782 \\ 4,3 & 415 \\ 4,3-4 & 44 \\ 4,6 & 362,724 \\ 4,6-8 & 723 \\ 4,7 & 290,296,362 \\ 4,8 & 362,724,813,829 \\ 4,9-13 & 379 \\ 4,16 & 762 \\ 4,17 & 389 \\ 4,18 & 848 \\ 4,19 & 762 \\ 4,21 & 408 \\ & \end{array}$

Der Brief an Titus

Brief 26, 156

1,14

$1,2408,734$

$1,5 \quad 786-787$

$1,7 \quad 701$ 


\begin{tabular}{|c|c|c|c|}
\hline 1,11 & 343,701 & 11,5 & 409,617 \\
\hline 1,13 & 832 & 11,7 & 284 \\
\hline 2,6 & 776 & 11,22 & 782 \\
\hline 2,13 & 734 & 11,38 & 617 \\
\hline 2,14 & 830 & 12,4 & 411 \\
\hline $3,3-5$ & $407,444-446$ & 12,20 & 617,782 \\
\hline $3,4-5$ & 398 & 12,27 & 434 \\
\hline 3,7 & 679,734 & 13,22 & 776 \\
\hline 3,8 & $361,445,774,831$ & & \\
\hline 3,10 & 402,454 & \multicolumn{2}{|c|}{ Der Brief des Jakobus } \\
\hline 3,11 & 697 & 1,11 & 359 \\
\hline 3,12 & $439-440$ & 1,24 & 438 \\
\hline \multirow[t]{2}{*}{3,13} & 832,834 & 3,1 & 826 \\
\hline & & 3,10 & 796 \\
\hline \multicolumn{2}{|c|}{ Der Brief an Philemon } & 3,17 & 393,405 \\
\hline Brief & $26,938-939,959-960,1014$ & & \\
\hline 1 & 4 & \multicolumn{2}{|c|}{ Der erste Brief des Petrus } \\
\hline 2 & 26 & 1,4 & 695 \\
\hline 4 & $420,425,427$ & 1,11 & 434 \\
\hline 8 & $422,783-786$ & 2,5 & 609 \\
\hline $8-9$ & 232 & 2,10 & 457 \\
\hline 9 & $4,784-785$ & 2,23 & 617 \\
\hline 10 & $784-785$ & 3,14 & 765 \\
\hline 11 & $407,446,518$ & 3,20 & 425 \\
\hline 12 & 580 & 4,1 & 735 \\
\hline \multirow[t]{2}{*}{13} & $694,762,774,784,786,830$ & 5,1 & 776 \\
\hline & $835-836,1014$ & 5,12 & 349 \\
\hline 14 & $694,766,784-786,836$ & & \\
\hline $15-16$ & 836 & \multicolumn{2}{|c|}{ Der zweite Brief des Petrus } \\
\hline 17 & $785-786,836$ & 1,13 & 430 \\
\hline 18 & 660 & 1,14 & 434 \\
\hline 19 & $784-785$ & 1,15 & 425 \\
\hline 20 & 762 & 2,10 & $743-744$ \\
\hline 21 & $694,733,786,836,1014$ & 3,4 & 432 \\
\hline 22 & $227,422,733$ & 3,5 & 770 \\
\hline \multicolumn{2}{|c|}{ Der Brief an die Hebräer } & \multicolumn{2}{|c|}{ Der erste Brief des Johannes } \\
\hline Brief & 341 & Brief & $40-41$ \\
\hline 3,13 & 410 & 2,19 & 322 \\
\hline 6,15 & 7,9 & 5,6 & 642 \\
\hline 7,2 & 405 & & \\
\hline 7,25 & 425 & \multicolumn{2}{|c|}{ Der zweite Brief des Johannes } \\
\hline 9,8 & 434 & \multirow{3}{*}{$\begin{array}{l}\text { Brief } \\
10\end{array}$} & $40-41$ \\
\hline 9,17 & 418 & & 747 \\
\hline 9,20 & 782 & & \\
\hline 9,28 & 735 & \multicolumn{2}{|c|}{ Der dritte Brief des Johannes } \\
\hline 10,27 & 720 & Brief & $40-41$ \\
\hline
\end{tabular}


Der Brief des Judas

$$
9 \quad 445,782
$$

Die Offenbarung des Johannes

Werk 6-15, 322, 394, 851-852

$2,7 \quad 361$

$2,8 \quad 351$

$2,10 \quad 852$

$5,7 \quad 319,365-366$

$6,2 \quad 670$

$6,4 \quad 670$

$7,11 \quad 322$

$7,14 \quad 319$

$8,5 \quad 319,321$

9,5

852

9,9
10,6
11,6
$12,7-8$
12,16
13,13
13,14
13,15
$13,16-17$
16,12
16,18
19,3
19,15
20,4
20,5

326

698

424

630

291

373

351

374

670

271

432

319

852

351

351

\section{Papyri und Inschriften}

4Q530

II, Z. 6-7 532

8HevXIIgr

XVI, Z. 25

Augustus

Res. Gest.

10

322

BGU

4.1147, Z. 18

206-208

Codex Boerniarianus

Papyrus $\quad 732$

\section{Griechisch-Römische Literatur}

Claudius Aelianus

De natura animalum

16.3

592

Achilleus Tatios Leucippe et Clitophon

5.3.8. 684
D

Papyrus $\quad$ 5, 532, 597

$\mathrm{P}^{46}$

Papyrus $\quad 349,609$

P.Hib.

1.53, Z. $1-2 \quad 311,368$

P.Petr.

2.2.2, Z. 5-6.9 311-312
Aristeasbrief

310

597

Aristoteles

Analytica priora

$34 \mathrm{a}$

323 
Physica

222b

845

Poetica

23

18

$1450 a \quad 44$

Athenaios

Deipnosophistae

581c 735

Chariton

De Chaerea et Callirhoe

5.10.2 695

Cicero

Autor

155

De inventione rhetorica

Werk

940

1.27

16

Dion Chrysostomos

Orationes

36.4

498

Diodor

Bibliotheca historica

13.34.6 787

Epiktet

Dissertationes

Werk 884

Epiphanius

Panarion

1.26

430

Eusebios

Praeparatio evangelica

11.10.2 845

Galenos

De facultatibus naturalibus

1.1

721
Herodot

Autor

330

Hesiod

Opera et dies

277

787

Homer

Ilias

1.64353

6.482-485 121

10.338-563 106

$13.730 \quad 358$

13.732-33 358

$16.19 \quad 838$

$20.306 \quad 353$

$22.158 \quad 301$

Odyssee

3.292-293

Horaz

Ars poetica

Werk

940

Isokrates

Archidamos

59

413

Josephus

Antiquitates judaicae

2.89

729

3.53

427

$4.91 \quad 301$

$4.249 \quad 787$

12.272-273 302

13.178-179 302

$15.140 \quad 427$

Bellum judaicum

1.27

427

Vita

Werk 961

$160 \quad 427$ 
Lysias

Orationes

1.24-25 319

12.5-12 338

Nikephoros

Vita sancti Andreae sali

$36 \quad 429-430$

Numenios Siehe Eusebius

Pastor Hermae

86.2

822

Pausanias

Periegesis

4.4.2-3 653

10.1.11 758-759

Philon

De aeternitate mundi

129

699

De fuga et inventione

38

729

De specialibus legibus

$\begin{array}{ll}1.290 & 725 \\ 2.79 & 787 \\ 2.225 & 669\end{array}$

Legatio ad Gaium

25

729

191

699

Quod omnis probus liber sit

132

729

Platon

Apologia

$32 \mathrm{a}-\mathrm{b} \quad 328$

34b 697

Gorgias

462e $\quad 750$

$510 \quad 681$
Leges

$767 a \quad 826$

$804 \mathrm{e} \quad 722$

Respublica

338c 681, 714

$361 \mathrm{C} \quad 413$

368a 721

Plinius der Jüngere

Autor $\quad 155$

Plutarch

Moralia

79a $\quad 826$

220b-c $\quad 324$

$274 \mathrm{C} \quad 759$

$577 a \quad 759$

$712 f \quad 847-848$

Polybios

Historiae

4.23.8 722

15.29.13 721

Pseudo-Clementinen

20.4.5 826

Quintilian

Institutio oratoria

$8.3 \quad 540$

Rhet. Her.

Werk 940

Sophokles

Ajax

768-769 721

Antigone

$452 \quad 435$

Theognis

Elegi

$329 \quad 356$ 
Theophrastos

De causis plantarum

4.1.10

597

Historia plantarum

7.1.7

597

Thukydides

Historiae

5.26

680-681

8.67 .2

413
Xenophon

Cyropaedia

4.2.1 382

$8.5 .10 \quad 330$

Hellenica

1.2.16 301

4.1.38 765 